This is an electronic reprint of the original article. This reprint may differ from the original in pagination and typographic detail.

Author(s): Kainulainen, Heikki; Papaioannou, Konstantinos G.; Silvennoinen, Mika; Autio, Reija; Saarela, Janne; Oliveira, Bernardo M.; Nyqvist, Miro; Pasternack, Arja; Hoen, Peter A.C. 't; Kujala, Urho; Ritvos, Olli; Hulmi, Juha

Title: $\quad$ Myostatin/activin blocking combined with exercise reconditions skeletal muscle expression profile of $\mathrm{mdx}$ mice

Year: $\quad 2015$

Version:

Please cite the original version:

Kainulainen, H., Papaioannou, K. G., Silvennoinen, M., Autio, R., Saarela, J., Oliveira, B. M., Nyqvist, M., Pasternack, A., Hoen, P. A. '., Kujala, U., Ritvos, O., \& Hulmi, J. (2015). Myostatin/activin blocking combined with exercise reconditions skeletal muscle expression profile of $\mathrm{mdx}$ mice. Molecular and cellular endocrinology, 399(January), 131-142. https://doi.org/10.1016/j.mce.2014.10.001

All material supplied via JYX is protected by copyright and other intellectual property rights, and duplication or sale of all or part of any of the repository collections is not permitted, except that material may be duplicated by you for your research use or educational purposes in electronic or print form. You must obtain permission for any other use. Electronic or print copies may not be offered, whether for sale or otherwise to anyone who is not an authorised user. 


\section{Myostatin/activin blocking combined with exercise reconditions skeletal muscle expression profile of mdx mice}

Heikki Kainulainen $^{\text {a }}$, Konstantinos G. Papaioannou ${ }^{\mathrm{a}}$, Mika Silvennoinen ${ }^{\mathrm{a}}$, Reija Autio ${ }^{\mathrm{b}}$, Janne Saarela $^{\mathrm{a}}$, Bernardo M. Oliveira ${ }^{\mathrm{a}}$, Miro Nyqvist ${ }^{\mathrm{c}}$, Arja Pasternack ${ }^{\mathrm{d}}$, Peter A.C. 't Hoen ${ }^{\mathrm{e}}$, Urho M. Kujala ${ }^{\text {, Olli Ritvos }}{ }^{\text {, }}$ Juha J. Hulmi ${ }^{\text {a }}$

${ }^{\mathrm{a} D e p a r t m e n t ~ o f ~ B i o l o g y ~ o f ~ P h y s i c a l ~ A c t i v i t y, ~ N e u r o m u s c u l a r ~ R e s e a r c h ~ C e n t e r, ~ U n i v e r s i t y ~ o f ~}$ Jyväskylä, Rautpohjankatu 8, P.O. Box 35, FI-40014 University of Jyväskylä, Finland

${ }^{b}$ Department of Signal Processing, Tampere University of Technology, Korkeakoulunkatu 1, P.O. BOX 553, FI-33101 Tampere, Finland

${ }^{c}$ Department of Medical Biochemistry and Genetics, University of Turku, Finland

${ }^{\mathrm{d} D e p a r t m e n t}$ of Bacteriology and Immunology, Haartman Institute, University of Helsinki, Haartmaninkatu 3 (P.O. Box 21) FIN-00014, University of Helsinki, Finland

${ }^{\mathrm{e}}$ Department of Human Genetics, Leiden University Medical Center (LUMC), Postzone S-04-P, PO Box 9600, 2300 RC Leiden, The Netherlands

${ }^{\mathrm{f}}$ Department of Health Sciences, University of Jyväskylä, Rautpohjankatu 8, P.O. Box 35, FI-40014 University of Jyväskylä, Finland

Correspondence to: Juha Hulmi

Department of Biology of Physical Activity

University of Jyväskylä

P.O. Box 35

40014 Jyväskylä, Finland

Tel. +358408327001

Fax. +35814617422

Email: juha.hulmi@jyu.fi

Running title: Transcriptomics of myostatin blocking and exercise 


\section{Abbreviations:}

AcR (in figures), see sActRIIB-Fc

Aqp4, aquaporin 4;

Atg $4 \mathrm{~b}$, autophagy related 4B cysteine peptidase;

ANOVA, analysis of variance;

BCAA, branched chain amino acid

Cont, PBS placebo treated control

DMD, Duchenne Muscular Dystrophy;

Ebna1bp2, EBNA1 binding protein 2;

EDL, extensor digitorum longus;

FASN, fatty acid synthase;

$\mathrm{FC}$, fold change

FDR, False Discovery Rate;

GAPDH, glyceraldehyde-3-phosphate dehydrogenase;

GSEA, gene set enrichment analysis;

IGF, insulin-like growth factor;

KEGG, Kyoto encyclopedia of genes and genomes;

mdx, muscular dystrophy caused by X chromosomal mutation, animal model of DMD;

MUP, major urinary protein;

NES, normalized enrichment score;

p, phosphorylated;

PBS, phosphate buffered saline (a placebo/vehicle);

PDK4, pyruvate dehydrogenase kinase 4;

PGC-1 $\alpha$, peroxisome proliferator-activated receptor gamma coactivator 1-alpha;

PVDF, polyvinylidene difluoride

Run, voluntary wheel running;

sActRIIB-Fc, soluble activin receptor fused to Fc domain of immunoglobulin G1;

STAT5, signal transducer and activator of transcription 5;

TCA cycle, tricarboxylic acid cycle (also known as Krebs cycle and citric acid cycle);

UCP3, uncoupling protein 3

wt, wild type 


\section{ABSTRACT}

Duchenne Muscular Dystrophy is characterized by muscle wasting and decreased aerobic metabolism. Exercise and blocking of myostatin/activin signaling may independently or combined counteract muscle wasting and dystrophies. The effects of myostatin/activin blocking using soluble activin receptor-Fc (sActRIIB-Fc) administration and wheel running were tested alone or in combination for seven weeks in dystrophic mdx mice. Expression microarray analysis revealed decreased aerobic metabolism in the gastrocnemius muscle of mdx mice compared to healthy mice. This was not due to reduced home-cage physical activity, and was further downregulated upon sActRIIB-Fc treatment in enlarged muscles. However, exercise activated pathways of aerobic metabolism and counteracted the negative effects of sActRIIB-Fc. Exercise and sActRIIB-Fc synergistically increased expression of major urinary protein, but exercise blocked sActRIIB-Fc induced phosphorylation of STAT5 in gastrocnemius muscle. In conclusion, exercise alone or in combination with myostatin/activin blocking corrects aerobic gene expression profiles of dystrophic muscle towards healthy wild type mice profiles.

Keywords: Physical activity, muscular dystrophy, muscle hypertrophy, mRNA profiling, oxidative metabolism 


\section{INTRODUCTION}

Duchenne Muscular Dystrophy (DMD) is a disease characterized by progressive wasting of skeletal muscle (Koenig et al 1987). Restoration of dystrophin expression in all of the muscles of the body is difficult, and the effectiveness of such treatment likely depends on the muscle quality of DMD patients. Therefore, approaches aimed at the stimulation of muscle growth and function are being developed, which may complement dystrophin restoration approaches (Amthor and Hoogaars 2012,Ljubicic et al 2014).

The lack or inhibition of myostatin seems to have various positive effects, such as increased muscle size and strength (Pistilli et al 2011,Amthor and Hoogaars 2012,Zhou et al 2010,Hulmi et al 2013a,Hoogaars et al 2012,McPherron and Lee 1997,Lee et al 2005). Recent evidence showed that it also increases muscle mass after a single-dose treatment in humans (Attie et al 2013), supporting its possible therapeutic use for some neuromuscular diseases and muscle wasting. However, there is also some evidence that it can have side-effects, such as decreased muscle oxidative capacity (Rahimov et al 2011,Amthor et al 2007,Matsakas et al 2012,Matsakas et al 2010,Hulmi et al 2013a,Ploquin et al 2012,Relizani et al 2014). On the other hand, muscular dystrophy is in itself associated with a decrease in oxidative capacity and gene expression in skeletal muscle (Timmons et al 2005). Low aerobic capacity, which is an outcome of decreased muscle oxidative metabolism, is a strong predictor of increased all-cause mortality (Blair et al 1989). Moreover, in DMD (Webster et al 1988) and in its animal model mdx mice (Moens et al 1993), less oxidative muscle fibers result in a decreased resistance to dystrophic pathology (for a review, see (Ljubicic et al 2014)). Thus, treatments that improve oxidative capacity may be especially beneficial to dystrophic muscle and are anticipated to complement myostatin blocking.

Some forms of exercise such as voluntary wheel running may offer benefits in mdx mice (Landisch et al 2008,Call et al 2008), whereas forced high intensity exercise, e.g. treadmill running, may 
sometimes be detrimental to dystrophic mice or humans (Grange and Call 2007). Recent evidence indicates that muscle endurance and markers of aerobic capacity seem to be positively affected by exercise when combined with myostatin blocking (LeBrasseur et al 2009,Hulmi et al 2013b) and after myostatin deletion (Matsakas et al 2012,Matsakas et al 2010). Timmons et al. (Timmons et al 2005) has identified approximately 90 genes in skeletal muscle that are responsive to endurance exercise training in healthy individuals and that are modulated similarly in DMD muscle when compared to healthy controls. An exception was a collection of genes involved in oxidative metabolism that were regulated oppositely in DMD and in exercise, i.e. upregulated by exercise, but downregulated by DMD (Timmons et al 2005). It is, however, not known whether exercise in dystrophic muscle with or without myostatin/activin blocking modulates gene expression pathways towards or away from the pattern observed in healthy muscle. Moreover, the possible interaction effects of these theoretically opposite treatments is interesting because it is known that the effect of drug or gene modulation can be different depending on whether it is administered to an active or sedentary individual (Booth and Laye 2009). On the other hand, the effects of exercise can depend on possible co-treatments. For instance, the positive effects of exercise on cardiorespiratory function and mitochondrial content were reported to be totally impaired when combined with simvastatin drug (Mikus et al 2013).

We have previously reported that running wheel exercise with or without myostatin/activin blocking in dystrophic mice increases the markers of oxidative capacity equal to or above the levels of healthy mice while normalizing the amount of voluntary running (Hulmi et al 2013b). Here, in a follow-up study, we provide a transcriptome-wide gene expression profiling of dystrophic murine skeletal muscle after exercise and/or myostatin/activin blocking. By simultaneously comparing these treatments to healthy control mice, we observed that exercise modulates dystrophic muscle towards a healthier profile in the gene sets of aerobic metabolism. Also independent, synergistic, 
compensatory and antagonistic effects of exercise and myostatin/activin blocking are shown in gene expression profiles as well as at the protein level.

\section{MATERIALS AND METHODS}

\subsection{Animals}

Six to seven weeks old male $\mathrm{C} 57 \mathrm{Bl} / 10 \mathrm{ScSnJ}$ mice and male mdx mice originating from the same strain (Jackson Laboratories, Bar Harbor, Maine, USA) were used in the experiments. The mice were housed in standard conditions (temperature $22^{\circ} \mathrm{C}$, light from 8:00 AM to 8:00 PM) and had free access to tap water and food pellets (R36, 4\% fat, 55.7\% carbohydrate, $18.5 \%$ protein, $3 \mathrm{kcal} / \mathrm{g}$, Labfor, Stockholm Sweden).

\subsection{Ethics Statement}

The treatment of the animals was in strict accordance with the European Convention for the Protection of Vertebrate Animals Used for Experimental and Other Scientific Purposes. The protocol was approved by the National Animal Experiment Board (Permit Number: ESLH-200908528/Ym-23).

\subsection{Experimental design}

Myostatin/activin blocker in the form of soluble activin receptor Fc (sActRIIB-Fc, 5-mg/kg) or placebo (PBS) was injected intraperitoneally once per week for seven weeks with or without voluntary wheel running exercise in young 7-week old $\mathrm{mdx}$ mice. Thus, the $\mathrm{mdx}$ mice were randomly divided into 4 groups in a 2 x 2 design: 1) sedentary control injected with PBS placebo, 2) sedentary injected with sActRIIB-Fc, 3) running wheel and injection with PBS placebo, and 4) running wheel with sActRIIB-Fc ( $\mathrm{n}=8$ in each). As a fifth group, wild type mice (C57B1/10ScSnJ) 
were injected with PBS and acted as healthy sedentary controls $(n=5)$. Thus the design included both wild type and mdx controls. To allow for the sActRIIB-Fc treatment to take effect, the mice were prevented from exercising by locking the running wheels for two days at the start of the experiment. In order to study only long-term effects of exercise, the mice also did not have access to running wheels on the last two days of the experiment.

During the experiments all conditions were standardized. The injections were conducted in pairs. One person prepared sActRIIB-Fc or PBS and searched for the next mouse while the other person performed the injection without knowing the identity of the mouse or the treatment. The investigators in every analysis were blinded to the treatment group. The mice were euthanized after the experiments by cervical dislocation after whichblood and tissue samples were collected.

\section{4. sActRIIB-Fc production}

The recombinant fusion protein was produced and purified in house as described in detail earlier (Hulmi et al 2013a). Our protein is similar, but not identical to that originally generated by Se-Jin Lee (Lee et al 2005). In short, the fusion protein contains the ectodomain (ecd) of human sActRIIB and a human IgG1 Fc domain. The protein was expressed in Chinese hamster ovary (CHO) cells grown in a suspension culture.

\subsection{Voluntary wheel running, feed intake and muscle function}

Voluntary wheel running was selected as the exercise modality in this study as it may offer benefits in mdx mice (Landisch et al 2008,Call et al 2008), whereas forced exercise, such as treadmill running, may sometimes be detrimental to dystrophic mice (Grange and Call 2007).

The mice were individually housed in cages where they had free access to custom-made running wheels (diameter $24 \mathrm{~cm}$, width $8 \mathrm{~cm}$ ) $24 \mathrm{~h} /$ day. Sedentary animals were housed in similar cages without the running wheel. 


\subsection{Home-cage activity by force plate recording}

Vertical ground reaction forces were measured using a custom-made force plate (linearity 99\%, cross-talk $<2 \%$ ). Forces were measured by four uniaxial strain gauges, one located in each corner of the cage. A 14-bit A/D converter (DI-710, DATAQ Instruments, Akron, OH, USA) was used for digitizing the force data at a sampling rate of $80 \mathrm{~Hz}$. The measurement range of the strain gauge was $\pm 250 \mathrm{~g}$, while the digitization precision was $<0.02 \mathrm{~g}$.

Three measurement time-points were selected: 1-5 days before the first treatment injections, during the third week of the treatments and at the end of the sixth week. Each measurement was conducted over a period of 24-hours. The measurement was conducted in the animal's own cage and in the same room with the other mice in this experiment. The recorded force values were summed from four strain gauges followed by filtering. The activity index was calculated as follows. The absolute values of the differences between consecutive force values were calculated. The mean of the absolute values were calculated from every second (20 values per second), the 1-second means were summed for $24 \mathrm{~h}$ period, and the sum was divided by the body mass of the mouse. The background value, calculated from the lowest activity index, was removed. The activity time calculation was based on identifying and summing the active seconds from the activity index data. These seconds were identified as active when the activity index value was higher than the threshold level (4 times the level of background activity). This activity analysis has been modified for our purposes from analysis methods previously developed and validated by us and others (Biesiadecki et al 1999,Rantalainen et al 2011,Silvennoinen et al 2014).

\subsection{Muscle sampling}

Hind leg muscles gastrocnemius, soleus, musculus quadriceps femoris, extensor digitorum longus (EDL) and tibialis anterior were immediately removed, weighed, and frozen in liquid nitrogen. The 
muscle weights have been published earlier (Hulmi et al 2013b). Gastrocnemius muscle was selected for further analysis.

\subsection{Western immunoblot analyses}

The gastrocnemius muscle was pulverized in liquid nitrogen and then $\sim 40 \mathrm{mg}$ of the powder was homogenized in ice-cold buffer with proper inhibitors and total protein measured using the bicinchonic acid protein assay (Pierce Biotechnology, Rockford, IL) with an automated KoneLab analyzer (Thermo Scientific, Vantaa, Finland) as previously reported (Hulmi et al 2013b).

Muscle homogenates in a Laemmli sample buffer were heated at $95 \mathrm{C}^{\circ}$ to denature proteins and further treated similarly as previously (Hulmi et al 2013b,Hulmi et al 2013a). In short, protein was separated by SDS-PAGE and transferred to a PVDF membrane, blocked and incubated overnight at $4^{\circ} \mathrm{C}$ with primary antibodies. The membrane was then washed and incubated with secondary antibodies (Jackson ImmunoResearch Laboratories, West Grove, PA, USA) for $1 \mathrm{~h}$ followed by washing. Proteins were visualized by ECL (SuperSignal west femto maximum sensitivity substrate, Pierce Biotechnology, Rockford, IL, USA) and quantified using a ChemiDoc XRS device with Quantity One software (version 4.6.3. Bio-Rad Laboratories, Hercules, CA, USA).

The uniformity of the protein loading was confirmed by staining the membrane with Ponceau $\mathrm{S}$ and by re-probing the membrane with an antibody against GAPDH (Abcam, Cambridge, UK). The results are corrected to the mean of Ponceau S (strong band at $\sim 42 \mathrm{kDa}$ ) and GAPDH value.

Antibodies. The antibodies recognized the phosphorylation of STAT5 at Tyr ${ }^{694}$, fatty acid synthase (FASN) (Cell Signaling Technology, Danvers, MA, USA), major urinary proteins (MUP), uncoupling protein 3 (UCP3) (Abcam, Cambridge, UK) and pyruvate dehydrogenase 4 (PDK4) (Novus Biologicals, Littleton, CO, USA). Moreover, STAT5 total protein was analyzed using specific antibody against STAT5 (Santa Cruz Biotechnology, Santa Cruz, CA, USA) with similar protocols as earlier (Hulmi et al 2013b). 


\subsection{RNA and DNA isolation and cDNA synthesis}

Gastrocnemius muscle was pulverized and homogenized in liquid nitrogen after which 65-75 mg of muscle powder was weighed into Trizol reagent (Invitrogen, Carlsbad, CA, USA). Total RNA and DNA fractions were extracted according to the manufacturer's guidelines and analyzed with Nanodrop ND-1000 (Thermo Fisher Scientific Inc., Waltham MA, USA) in duplicate. Before mRNA analysis, any possible remaining DNA was further degraded using DNAse kit (TURBO DNA-free ${ }^{\mathrm{TM}}$ Kit, Applied Biosystems by Life Technologies, South San Francisco, CA, USA). The quality of RNA was confirmed by spectrophotometry $\left(\mathrm{OD}_{260} / \mathrm{OD}_{280}\right.$ ratio of 1.9 to 2.0$)$, agarose gel electrophoresis as well as using Agilent Bioanalyzer (Agilent Technologies Inc., Santa Clara, CA, USA). From RNA-gels stained with ethidiumbromide, $18 \mathrm{~S}$ and $28 \mathrm{~S}$ ribosomal RNA (rRNA) were photographed under UV-light and quantified using a ChemiDoc XRS device with Quantity One software (version 4.6.3. Bio-Rad Laboratories, USA).

\subsection{Microarray analysis}

RNA samples of 5 mice from each group and 4 from the controls were analyzed with Illumina Sentrix MouseRef-8 v2 Expression BeadChip containing approximately 25600 annotated transcripts and over 17900 unique genes (Illumina Inc., San Diego, CA, USA) by the Finnish Microarray and Sequencing Centre at the Turku Centre for Biotechnology according to the manufacturer's instructions. Raw data were normalized with quantile normalization (including log2-transformation of the data) (Smyth and Speed 2003) and data quality was assessed using Chipster software (IT Center for Science, Espoo, Finland) (Kallio et al 2011) and R with Limma package (Smyth 2004). The normalized gene expression data were imported into Excel spreadsheets (Microsoft Corp., Redmond, WA, USA). From the genes represented by multiple probes, the probe with the highest fold-change ratio was used for further analysis. MIAME guidelines were followed during array data generation, preprocessing, and analysis. The complete data set is publicly available in the NCBI Gene Expression Omnibus (http://www.ncbi.nlm.nih.gov/geo/; accession no. GSE52766). 


\subsection{Data Analysis}

The differentially expressed genes in the microarray data between the mdx and wild type sample groups were detected utilizing linear modeling and empirical Bayes methods of the Limma package of $\mathrm{R}$ while the raw p-values were adjusted using the Benjamini and Hochberg method. The genes with an adjusted $\mathrm{P} \leq 0.05$ and with absolute fold change $\geq 1.5$ were considered to be differentially expressed.

Enrichment of functionally related genes in three different gene set collections was first performed using a non-biased method by Gene Set Enrichment Analysis software (GSEA; Version 2.0) (57) as previously done in our laboratory (Leskinen et al 2010,Kivela et al 2010,Kujala et al 2013). For this analysis, ranking lists representing different ratio combinations of the normalized data of the five experimental groups were created by averaging the group results for each gene. The fold-change ratio was the chosen method to rank the genes to discover enriched gene sets. The collection used was the Canonical Pathways collection (1452 gene sets, C2:CP, version 3.0) (http://www.broadinstitute.org/gsea/msigdb/collections.jsp). The number of permutations by gene set was set to 1000 and gene sets with at least five and no more than 500 genes were taken into account in each analysis. Each analysis was carried out at least five times and all the results were averaged into a single value. The statistical significance was calculated using false discovery rate (FDR). The level of significance was set at FDR $<0.05$. The DAVID functional annotation (Dennis et al 2003) was used to further analyze the effects of the phenotype and the treatment, and the genes upregulated with fold change $\geq 1.5$ were uploaded to DAVID. GSEA is able to reveal changes in expression of a large set of genes even when the average change of gene expression is $20 \%$ as typically is the case with e.g. oxidative metabolism (Mootha et al 2003, Subramanian et al 2005), and even lower when an adaptation effect of exercise is investigated (Egan and Zierath 2013). In DAVID, the enrichment analysis was run against the default gene groups including gene ontologies (Ashburner et al 2000), and KEGG-pathways (Kanehisa et al 2008), as well as against chromosomal 
locations and cytoband information. Heatmap illustrations were performed with MATLAB, and the across-samples standardized gene expression data was hierarchically clustered utilizing Euclidean distance and average linkage methods.

All the other data except microarray were evaluated by analysis of variance (ANOVA) followed by Tukey's post hoc test. The level of significance in these analyses was set at $p<0.05$. Data are expressed as means \pm SE. Correlations were analyzed using Pearson's Product Moment Coefficient. 


\section{RESULTS}

Myostatin / activin blocker in a form of soluble activin receptor Fc (sActRIIB-Fc) or placebo (PBS) was injected seven weeks with or without voluntary running exercise in young male mdx mice. Wild type male $\mathrm{C} 57 \mathrm{Bl} / 10 \mathrm{ScSnJ}$ mice, originating from the same strain as mdx mice, were injected with PBS and acted as healthy non-exercised controls.

\subsection{Physiological characteristics}

This is a follow-up study of (Hulmi et al 2013b). In brief, sActRIIB-Fc increased muscle mass in all the muscles weighed whereas exercise in these mdx mice enhanced muscle aerobic capacity (e.g. citrate synthase and SDH activities) to similar or higher levels than those observed in healthy mice without major changes in muscle histology. With the exception of the first 2-3 weeks of fast muscle growth when the volume of voluntary running, and capillary and mitochondrial density were

decreased by sActRIIB-Fc, no major effect of sActRIIB-Fc on the determinants or regulators of oxidative metabolism were observed as explained earlier (Hulmi et al 2013b). Previously unreported relative comparisons of the effect of sActRIIB-Fc in increasing muscle mass in sedentary (sActRIIB-Fc vs. PBS) or in running mice (sActRIIB-Fc running vs. PBS running) were as follows (mean $\pm \mathrm{SE}$ ): gastrocnemius $26.7 \pm 6.3 \%$ and $13.7 \pm 4.3 \%$ (difference between sedentary and running mice injected with sActRIIB-Fc: $\mathrm{P}<0.05$ ), soleus $10.1 \pm 4.2 \%$ and $16.5 \pm$ $6.1 \%$ (NS.), EDL $19.5 \pm 5.4$ and $19.9 \pm 4.8 \%$ (NS.), tibialis anterior $25.3 \pm 4.5$ and $14.0 \pm 4.2 \%$ ( $\mathrm{P}=0.11, \mathrm{NS}$.$) , quadriceps 25.8 \pm 4.1 \%$ and $15.5 \pm 3.4 \%(\mathrm{P}=0.09$, NS.).

\subsection{Muscle ribosomal RNA is increased in mdx mice and sActRIIB-Fc decreases muscle DNA content}

Higher total RNA concentration per mg of muscle tissue was observed in mdx mice when compared to similarly treated healthy wild type mice, without any effect of running or sActRIIB-Fc (Fig. 1A). Concordantly, 28S and 18S rRNA bands had 20-50\% higher intensity in mdx control than in wild 
type control mice with a tendency for running to further increase $28 \mathrm{~S}$ and $18 \mathrm{~S}$ RNA by 25 ( $\mathrm{P}=0.06)$ and $10 \%(\mathrm{P}=0.10)$, respectively without an effect of sActRIIB-Fc (Fig. 1B). Muscle protein concentrations were unaffected by dystrophy or the treatments (Fig. 1C). sActRIIB-Fc administration decreased muscle DNA concentrations (ANOVA $\mathrm{P}=0.03$ ) the direction being towards wild type mice (Fig. 1D), whereas exercise decreased the ratio of protein to RNA (ANOVA $\mathrm{P}=0.01$ ), the direction being slightly away from that of wild type mice (1E). The ratio of protein to DNA was decreased in mdx mice when compared to wild type mice, but this difference was prevented by the combination of running and sActRIIB-Fc (Fig. 1F).

\subsection{Gene expression profile of mdx mice is corrected towards healthy mice by the treatments}

Clustering the whole gene expression data revealed clear differences between the mdx and wild type control mice. Instead, there was no clear division between the various differently treated mdx samples in the genome-wide clustering result (Supplemental Fig S1). Next, the calculation of the number of individual genes toward or away from the level of controls was conducted using a $50 \%$ distance criteria. All the treatments tended to slightly modulate the gene expression profile of the gastrocnemius muscle towards that of healthy muscle and the effect tended to be strongest for exercise or the combination of exercise and sActRIIB-Fc (Table 1).

\subsection{The modulation of gene sets towards wild type mice with exercise}

The main purpose of the present study was to investigate overall trends in larger gene sets instead of single genes as well as the adaptation of muscle transcriptome to exercise training, a response that is typically small in the individual mRNA levels (Egan and Zierath 2013), as in the present study with running wheels. Considering that also the timing of muscle collection was after many weeks of rather moderate doses of sActRIIB-Fc administration instead the onset of higher dose treatment (Hulmi et al 2013a) and that there is a rather large variation within mdx mice, no consistent changes in individual genes were observed in the microarray due to sActRIIB-Fc (the genes most responsive are listed as a supplemental Table S1). A further mRNA analysis was thus conducted using gene 
clustering analysis from the gastrocnemius muscle with a gene set enrichment analysis (GSEA) that is designed to find physiologically meaningful results from large gene sets with $<20 \%$ average changes in individual gene expression (Subramanian et al 2005). Analysis using DAVID produced, to a large extent, similar results and thus the results from GSEA are shown if not otherwise mentioned. Of the canonical processes or pathways, 212 were upregulated and 24 downregulated between mdx mice and wild type control mice both injected with PBS placebo at FDR $<0.05$ (Supplemental Table S2). Most notably, pathways for aerobic metabolism (e.g. electron transport chain, metabolism of lipids and lipoproteins, branched chain amino acid (BCAA) degradation, oxidative phosphorylation) were among the 10 most downregulated pathways in dystrophic muscle in GSEA (FDR $<0.005$, Fig. 2 and Supplemental Table S2) while the oxidative mitochondrial pathways were also the most downregulated in DAVID (Supplemental Table S4). Decreases in these pathways were associated with the downregulation of peroxisome proliferator-activated receptor gamma coactivator 1-alpha (PGC-1 $\alpha$ ) pathway gene set in $\mathrm{mdx}$ mice when compared to wild type mice (Biocarta, FDR=0.01) (Supplemental Table S2) along with the previously reported decreased PGC-1 $\alpha$ protein in these mice (Hulmi et al 2013b). sActRIIB-Fc treatment alone decreased some of these (electron transport chain and oxidative phosphorylation) even further $($ FDR $<0.05)$ (Fig. 2A-B green spheres). Importantly, all of the most strongly downregulated pathways that were mentioned above, as well as many others downregulated in mdx mice (e.g. fatty acid metabolism and glucose regulation for insulin secretion), were upregulated by aerobic exercise and thus towards the wild type controls (Fig. 2). More results of the pathways in different comparisons are shown in Supplemental Table S2. The genes contributing to the enrichments of these gene sets are shown in Supplemental Table S3.

Supporting the GSEA results, DAVID analysis showed that individual genes from a gene set of lipid metabolism revealed a profile in which several genes regulating lipid metabolism were downregulated in dystrophic muscle and this difference gradually disappeared with exercise in most 
of the mice (Fig. 3). In summary, running modulated dystrophic muscle towards a profile of wild type mice in the level of absolute number of genes and gene sets of aerobic energy metabolism. Myostatin/activin blocking downregulated some of the gene sets for mitochondrial energy production, but exercise attenuated or blocked this decrease.

\subsection{Myostatin/activin blocking attenuated some of the exercise-effects on aerobic metabolism}

Many of the effects of exercise on gene sets were similar whether sActRIIB-Fc treatment was used or not. This can be seen in similar increases in e.g. electron transport chain, oxidative phosphorylation, BCAA degradation, fatty acid oxidation, peroxisome as well as metabolism of lipids and lipoproteins in the GSEA analysis (red and yellow spheres in Fig. 2, respectively). However, differences were also observed. For instance, exercise alone (red spheres in Fig. 2) significantly increased tricarboxylic acid $(\mathrm{TCA})(\mathrm{FDR}=0.003)$ and glucose regulation of insulin secretion $(F D R=0.002)$ pathways, but sActRIIB-Fc co-treatment blocked these exercise effects (yellow spheres in Fig. $2 \mathrm{C}$ and $2 \mathrm{G}, \mathrm{FDR}=0.59$ and $\mathrm{FDR}=0.18$, respectively).

\subsection{More gene sets are differently regulated with combined treatment compared to single treatment alone}

GSEA showed that sActRIIB-Fc activated 92 canonical cell processes in active mice (mdxsActRIIB-Fc Run vs. mdx-Run), but only one process in sedentary mice (mdx-sActRIIB-Fc vs. mdx-Cont) $($ FDR $<0.05)$ (Supplemental Table S2). Furthermore, sActRIIB-Fc did not decrease any canonical cell process in active muscles, but decreased 11 of these processes in sedentary muscle $($ FDR $<0.05)$ (Supplemental Table S2). Exercise activated 35 canonical cell processes in mice treated with sActRIIB-Fc (mdx-sActRIIB-Fc Run vs. mdx-sActRIIB-Fc), but only 19 processes in PBS-treated mice (mdx-Run vs. mdx-control) $($ FDR $<0.05)$ (Supplemental Table S2). Exercise downregulated $(\mathrm{FDR}<0.05)$ only one canonical cell process in mice treated with sActRIIB-Fc, but 17 processes in PBS-treated mice (Supplemental Table S2). Therefore, the effects of sActRIIB-Fc 
and exercise were different in mice subjected to combined treatments as compared with animals given either treatment alone.

\subsection{Various proteins are independently regulated or interact due to the effects of myostatin/activin blocking and exercise}

To further understand the possible independent and interaction effect of these two treatments, we analyzed several proteins or phosphoproteins that have a role in energy metabolism and/or proteins of which gene expression were affected in microarray by the treatments or the phenotype. sActRIIB-Fc increased the phosphorylation of STAT5 only in sedentary muscle $(\mathrm{P}<0.01$ and exercise $\mathrm{x}$ drug interaction effect $\mathrm{P}=0.048$ ) (Fig. 4A). Major urinary protein (MUP), a lipocalin family of proteins also involved in energy metabolism (Hui et al 2009), tended to be enriched by exercise in microarray (DAVID, not shown) and show changes between the groups in individual MUP genes (Supplemental Fig. S2). More important, there was an exercise $\mathrm{x}$ sActRIB-Fc interaction effect in MUP ( $\mathrm{P}=0.009)$ (Fig. 4B). This was observed as increased MUP with combined treatment (sActRIIB running) when compared to running alone $(\mathrm{P}<0.05)$. Lipid metabolism was downregulated in mdx mice and activated by exercise as shown in Fig. 2 and Fig. 3. The effect of exercise on this gene set is supported by increased protein content for protein important in glucosefat switch, PDK4 (running effect $\mathrm{P}=0.004$ ), independent of sActRIIB-Fc (Fig. 4C, Supplemental Fig. S2). The level of PDK4 was unchanged after 7 weeks of sActRIIB-Fc injection, but it was significantly decreased $\sim 30 \% 2$ days after sActRIIB-Fc (Supplemental Fig. S3) showing that myostatin blocking can regulate PDK4, but this effect seems to be diminished after longer-term (2-7 weeks) treatment. In another protein regulating energy metabolism, UCP3, a trend for an increase was observed in PBS-treated mice $(\mathrm{P}=0.09)$ (Fig. 4D). Another protein that has a role in muscle lipid metabolism, FASN, was not responsive to any of the treatments (Fig. 4E). A clear increase in the overall expression was seen for phosphorylated and total ERK 1/2 in mdx mice when compared 
to wild type control mice $(\mathrm{P}<0.05)$ without an effect of the treatments or changes in the ratio of pERK $1 / 2$ to ERK 1/2 (Fig. 4F). These results showed that sActRIIB-Fc and exercise strongly interacted with regard to MUP and pSTAT5, whereas the effect of exercise on PDK4 was independent of sActRIIB-Fc.

\subsection{Exercise and sActRIIB-Fc effects at the mRNA level}

Although the focus of the present study was gene sets instead of individual genes, genes with high expression levels in our micro array due to the treatments and/or phenotype and/or previously shown to have a function in exercise or growth were selected for analysis by quantitative Real-Time PCR (See Supplemental Methods). Of those, aquaporin 4 mRNA, a water-channel protein (Basco et al 2013), increased due to exercise $(2 \times 2$ ANOVA exercise effect $\mathrm{P}=0.002)$, in a similar way as the in microarray data (increased in every running vs. sedentary mice, but the mean change $32 \%$ was not significant after multiple correction, not shown), restoring the difference towards the wildtype control levels (Supplemental Fig S4). Dystrophic mice had $50 \%$ lower levels of myostatin mRNA than wildtype controls $(\mathrm{P}<0.001)$ (Supplemental Fig S4) suggesting that the effect of blocking myostatin in mdx mice may be weaker than in healthy mice. No effects of the treatments or muscle dystrophy were observed on Atg4b or on Ebna1bp2 mRNA (Supplemental Fig S4) or on the housekeeping gene GAPDH relative to total RNA.

\subsection{Home-cage habitual physical activity}

To examine whether voluntary activity levels may explain differences in the oxidative gene signature of dystrophic and wild type mice as well as sActRIIB-Fc and PBS-injected mice, habitual physical (locomotor) activity was analyzed. Before the treatments began, no differences were observed between the different groups of mdx mice. During the experiment, there was a significant group-effect observed in a 2 factor repeated measures ANOVA in both activity time and activity index, which was explained simply by the groups with running wheel access being more active. No 
consistent differences in the measures of the level of habitual activity between mdx and wild type mice were observed, except at 6 weeks ( $\sim 13$ weeks of age) when the activity time of mdx mice was slightly higher when compared to control mice (Fig. 5). Thus, it seems that lower physical activity levels cannot explain the decreased aerobic metabolism in mdx mice. In addition, no change was observed in sActRIIB-Fc injected mice, thus the effects of sActRIIB-Fc on further decreasing gene sets of oxidative phosphorylation and the electron transport chain were probably direct and not through changes in physical activity.

To further confirm that physical activity does not explain lower gene expression activity of oxidative metabolism and related gene sets in mdx mice, Pearson's correlation coefficients were calculated between the physical activity measures and several signaling pathway centroids (Kivela et al 2010) for PBS-injected mdx and wild type control groups separately and together $(n=9)$. Signaling pathways selected for this analysis were ones that were significantly different in both mdx vs. wild type and running vs. non-running comparisons. Centroids were calculated as a mean of enriched or leading-edge subset of genes (i.e. genes producing the GSEA enrichment score) of a certain signaling pathway. The genes contributing to the enrichment of these gene sets are presented in the Supplemental Table S3. The activity time or index did not correlate significantly $(\mathrm{P}>0.05)$ between any of the centroids and, thus, cannot explain the decreased aerobic metabolism in the mdx mice (Supplemental Table S5).

\section{DISCUSSION}

In this follow-up study (Hulmi et al 2013b) we point out that the further decrease in oxidative capacity by myostatin/activin blocking, also reported by others (Rahimov et al 2011,Amthor et al 
2007,Matsakas et al 2012,Matsakas et al 2010,Hulmi et al 2013a,Ploquin et al 2012,Relizani et al 2014), is localized to the gene sets of oxidative phosphorylation and the electron transport chain. These two pathways along with other pathways regulating oxidative metabolism were, however, upregulated by aerobic exercise to similar or even higher level than in healthy wild type controls. This finding is significant considering the importance of aerobic capacity and aerobic muscle phenotype in health (Blair et al 1989,Kujala et al 1998). Furthermore, this is of interest especially in muscular dystrophy, as the role of low oxidative capacity in the pathophysiology of mdx and DMD muscles is well established (Timmons et al 2005,Kuznetsov et al 1998,Hulmi et al 2013b,Moens et al 1993, Webster et al 1988,Ljubicic et al 2014).

The reason for the remodeling of muscle dystrophic muscle fibers towards less oxidative phenotype, also noticed earlier (Zhao et al 2009,Rahimov et al 2011,Chelh et al 2009), is unknown. It has been suggested that a possibly lower activity level in muscular dystrophy could explain, at least in part this result and thus may be an indirect effect of the disease. The voluntary running levels in running wheels (Hara et al 2002) and also locomotion activity of mdx mice have been reported to be lower than that of wild type mice (Landisch et al 2008). We have unpublished evidence that, indeed, the distance travelled in our mdx mice was lower than in wildtype mice before the start of the treatments (at the age $\sim 6$ weeks), but not 3 or 6 weeks later. However, our data measured with a force plate system (Silvennoinen et al 2014), which takes into account all forces acting against the ground including stationary movements (e.g. eating, drinking and grooming), showed that the level of habitual home-cage physical activity was not lower in mdx mice. Furthermore, correlation analysis showed that habitual activity did not explain the major differences in the gene sets of aerobic metabolism observed between $\mathrm{mdx}$ and wild type control mice. We cannot exclude the idea that some forms of lower intensity physical activity that were not detected with our system might explain the decreased aerobic metabolism. Nevertheless, our results suggest that the main reason for the decreased aerobic metabolism is likely something other than the lower level of physical activity 
of mdx mice. The lack of dystrophin has a role in impaired signaling at the sarcolemma (Evans et al 2009). One possible mediator of decreased aerobic capacity/metabolism in mdx muscle and its restoration by running is $\mathrm{PGC}-1 \alpha$, probably the best-known regulator of oxidative capacity (Chinsomboon et al 2009). We previously reported a decrease in PGC-1 $\alpha$ protein in the gastrocnemius muscle of these 13-14 week old mdx mice, and the restoration of PGC-1 $\alpha$ to or above the wild type mice with running (Hulmi et al 2013b). In contrast, in the tibialis anterior muscle of mdx mice, an increased PGC-1 $\alpha$ protein has been reported suggesting a compensatory adaptation to minimize the extent of pathological changes in muscle (Ljubicic et al 2011). Nevertheless, in the present study, we provide further evidence using gene set enrichment analysis that the PGC-1 $\alpha$ pathway may be a strong candidate to explain the decreased aerobic metabolism in mdx mice and that this is independent of the habitual home-cage physical activity of these mice.

Unlike habitual physical activity, physical exercise in the form of wheel running had positive effects on oxidative metabolism in mdx mice. This supports our previous results showing that the activity, content and regulators of mitochondrial enzymes in the skeletal muscle of these mice were increased by running (Hulmi et al 2013b). Here, we show that higher expression could be seen in a wide range of gene sets from lipid and BCAA metabolism to the TCA cycle, electron transport and oxidative phosphorylation. These longitudinal results in mice support those of an earlier crosssectional study by Timmons et al. (Timmons et al 2005) who showed that various genes of oxidative phosphorylation acted in an opposite manner in subjects with DMD and healthy, previously sedentary people after a training period. Importantly, in the present study exercise was sufficient to block this decrease in oxidative metabolism and thus preserved the oxidative phenotype of muscle. This further supports the beneficial effect of exercise in at least mild forms of muscle dystrophy. A recent randomized controlled trial showed that well designed exercise is both feasible and safe and seems to reduce the functional deterioration due to muscle disuse in boys with DMD (Jansen et al 2013). Further studies are needed to investigate the dose and the type of exercise 
as certain types of heavy exercise, even though possibly beneficial in correcting aerobic metabolism, may sometimes actually be detrimental in muscular dystrophy patients (Grange and Call 2007). Even though there was a clear beneficial effects of exercise correcting the decreased aerobic metabolism in mdx mice, we did not previously notice in these mice any major improvements in mdx muscle histology or in muscle endurance after running, although increased daily voluntary running during the first weeks of the experiment suggests some form of improvement in more specific muscle function (Hulmi et al 2013b). Therefore, the effects of exercise should be investigated in the future together with dystrophin correction (Hoogaars et al 2012).

The expression of the gene set for BCAA degradation was previously reported to be associated with high aerobic capacity (Kivela et al 2010). The gene set of BCAA degradation is also increased in habitually physically active twin pairs compared with their inactive co-twins (Leskinen et al 2010) and is associated with lower serum BCAA concentration (Kujala et al 2013). In the present study, dystrophic mice had downregulated BCAA degradation that was reversed to a more or less healthy control level by exercise. This may reflect enhanced mitochondrial fatty acid oxidation and energy production (Kainulainen et al 2013,Wagenmakers 1998). Similarly to BCAA degradation, decreased gene sets of lipid and fatty acid metabolism in dystrophic muscle were observed supporting the previously reported impaired fat oxidation in $\mathrm{mdx}$ mice (Even et al 1994). Importantly, exercise increased and, thus, restored the gene sets of lipid and fatty acid metabolism in mdx mice. Increased fat metabolism by exercise was associated with increased PDK4 protein, a positive regulator of fat vs. glucose use as an energy source (Sugden and Holness 2003). Similarly to gene sets of fat metabolism, this increase also occurred independent of sActRIIB-Fc.

The second aim of the study was to investigate if the exercise or myostatin/activin blocking differentially affect muscle metabolic pathways when these treatment modalities are combined. In the present study we have shown independent, synergistic, compensatory and antagonistic effects of exercise and sActRIIB-Fc at the RNA and protein level. For instance, increases in some of the gene 
sets and protein levels by exercise were independent of sActRIIB-Fc (e.g. BCAA degradation and gene sets related to fatty acid and lipid metabolism, PDK4 and AQP4). On the other hand, exercise and sActRIIB-Fc acted in an opposing manners in the gene sets of electron transport chain and oxidative phosphorylation, with the effect of exercise being sufficient to block the sActRIIB-Fcinduced decreases. As an example of antagonistic effects, sActRIIB-Fc was able to block the upregulation in the TCA-cycle gene set induced by exercise while exercise was able to completely block the strongly induced phosphorylation of STAT5 by sActRIIB-Fc. A synergistic effect of exercise and sActRIIB-Fc was observed in MUP protein levels.

MUPs are thought to be produced mainly in the murine liver, but are also involved in the biogenesis of mitochondria (Hui et al 2009). In addition to the interaction effect of sActRIIB-Fc and exercise, elevated MUP was also observed in mdx mice when compared to wild type mice. Previously, in a proteomic analysis, MUPs were shown to be elevated in the plasma of mdx mice when compared to wild type mice (Colussi et al 2010). Clearly, more research is needed to understand the potential importance of MUPs in skeletal muscle. Another example that showed an interaction effect of exercise and sActRIIB-Fc was the phosphorylation of STAT5. In skeletal muscle, the transcription factor STAT5 conveys signals from hormones such as growth hormone and insulin and its deficiency leads to the downregulation of insulin-like growth factor I (IGF-I) expression (Klover and Hennighausen 2007, Chen et al 1997). Previously, IGF-I was shown to be mandatory for the hypertrophy induced by the overexpression of follistatin, a myostatin/activin blocker (Kalista et al 2012). Indeed, in the present study, exercise that blocked the phospho-STAT5 response also attenuated the sActRIIB-Fc-induced increase in gastrocnemius muscle size. The consequences of exercise in modulating the effects of myostatin/activin blocking warrants further mechanistic studies. Another protein possibly mediating some of the effects of exercise in dystrophic mice is a water-channel protein AQP4, which is associated with the dystrophin complex and its levels are reduced in DMD in humans and in mice (Nicchia et al 2008,Frigeri et al 2002). In the present study, 
the gene expression of AQP4 was induced by exercise in mdx mice restoring the difference to the level of wildtype controls. The functional importance of this exercise effect in dystrophic mice remains to be determined, but AQP4 has been recently shown to be important in exercise-induced adaptation of skeletal muscle of healthy mice (Basco et al 2013).

Previously, the effect of genes in various loss or gain of function experiments has been shown to be dependent on the exercise status (Booth and Laye 2009). On the other hand, the effects of exercise can depend on co-treatments, which supports our data. For instance, the effects of exercise on cardiorespiratory function and mitochondrial content were reported to be totally impaired when simvastatin drug was combined with exercise (Mikus et al 2013). The gene set enrichment analysis showed that the effect of sActRIIB-Fc was, in general, much larger in the exercised than in sedentary mice. The effect of exercise was also more pronounced in the sActRIIB-Fc-treated mice than in PBS-treated mice. Some of the effects of sActRIIB-Fc are similar to those of resistance training and different than those of aerobic training, such as increased muscle size and unaltered or even decreased aerobic capacity (Rahimov et al 2011,Mero et al 2013,Hulmi et al 2013b). It has been shown that the effects of aerobic exercise are more robust in resistance-trained than in endurance-trained muscle and vice versa (Coffey et al 2006). Thus as in the present study, the observed effect of sActRIIB-Fc (mimicking some effects of resistance exercise) can be stronger during co-treatment with endurance exercise when compared to single treatments. It is acknowledged that a medical treatment regulating muscle metabolism can also affect exercise activity levels. We showed earlier that sActRIIB-Fc decreases the amount of voluntary running in the first, but not anymore during last weeks of the experiment (Hulmi et al 2013b), similarly as what has been observed in myostatin depleted mice (Personius et al 2010).

Treatment with sActRIIB-Fc decreased muscle DNA concentration in mdx mice, and the direction of this change was towards the wild type control mice without an effect of the treatments on muscle RNA concentrations. This result after pharmacological blockade of myostatin and activins supports 
the earlier finding from myostatin depleted mice that were reported to have decreased DNA per mg muscle due to increased muscle mass, but unchanged RNA or protein concentrations (Welle et al 2006). Total protein per DNA was decreased in mdx mice when compared to wild type mice, but this difference was prevented by the combination of running and sActRIIB-Fc. The importance of these results in practice remains to be determined. Finally, it is possible that some other muscles, such as more oxidative soleus or diaphragm might have shown slightly different results, but we believe that the adaptations presented from gastrocnemius muscle reflect similar responses from the other muscles as well.

In summary, exercise can modulate gene expression profiles of a dystrophic gastrocnemius muscle towards healthy control mice regarding gene sets of oxidative metabolism. As low oxidative metabolism is involved in the pathophysiology of $\mathrm{mdx}$ and DMD muscles, exercise can be suggested to constitute a complementary therapy for dystrophin restoration approaches and treatment modalities for enhancing muscle strength and size. The level of habitual physical activity does not alone seem to explain the downregulated oxidative phenotype in mdx muscle and instead a direct effect of the lack of dystrophin is a more probable cause. The effects of exercise and blocking myostatin/activin can be in some measures similar, and in some different when the treatments are combined in comparison to the single treatments alone. Our present results support the recommendation to recognize the possible combined effect of physical activity or exercise, when studying the effects of a drug. 


\section{REFERENCES}

Amthor, H., Hoogaars, W.M., 2012. Interference with Myostatin/ActRIIB Signaling as a Therapeutic Strategy for Duchenne Muscular Dystrophy. Curr. Gene Ther. 12, 245-259.

Amthor, H., Macharia, R., Navarrete, R., Schuelke, M., Brown, S.C., Otto, A., Voit, T., Muntoni, F., Vrbova, G., Partridge, T., Zammit, P., Bunger, L., Patel, K., 2007. Lack of myostatin results in excessive muscle growth but impaired force generation. Proc. Natl. Acad. Sci. U. S. A. 104, 18351840.

Ashburner, M., Ball, C.A., Blake, J.A., Botstein, D., Butler, H., Cherry, J.M., Davis, A.P., Dolinski, K., Dwight, S.S., Eppig, J.T., Harris, M.A., Hill, D.P., Issel-Tarver, L., Kasarskis, A., Lewis, S., Matese, J.C., Richardson, J.E., Ringwald, M., Rubin, G.M., Sherlock, G., 2000. Gene ontology: tool for the unification of biology. The Gene Ontology Consortium. Nat. Genet. 25, 25-29.

Attie, K.M., Borgstein, N.G., Yang, Y., Condon, C.H., Wilson, D.M., Pearsall, A.E., Kumar, R., Willins, D.A., Seehra, J.S., Sherman, M.L., 2013. A single ascending-dose study of muscle regulator ace-031 in healthy volunteers. Muscle Nerve 47, 416-423.

Basco, D., Blaauw, B., Pisani, F., Sparaneo, A., Nicchia, G.P., Mola, M.G., Reggiani, C., Svelto, M., Frigeri, A., 2013. AQP4-dependent water transport plays a functional role in exercise-induced skeletal muscle adaptations. PLoS One 8, e58712.

Biesiadecki, B.J., Brand, P.H., Koch, L.G., Britton, S.L., 1999. A gravimetric method for the measurement of total spontaneous activity in rats. Proc. Soc. Exp. Biol. Med. 222, 65-69.

Blair, S.N., Kohl, H.W.,3rd, Paffenbarger, R.S.,Jr, Clark, D.G., Cooper, K.H., Gibbons, L.W., 1989. Physical fitness and all-cause mortality. A prospective study of healthy men and women. JAMA 262, 2395-2401.

Booth, F.W., Laye, M.J., 2009. Lack of adequate appreciation of physical exercise's complexities can pre-empt appropriate design and interpretation in scientific discovery. J. Physiol. 587, 55275539.

Call, J.A., Voelker, K.A., Wolff, A.V., McMillan, R.P., Evans, N.P., Hulver, M.W., Talmadge, R.J., Grange, R.W., 2008. Endurance capacity in maturing mdx mice is markedly enhanced by combined voluntary wheel running and green tea extract. J. Appl. Physiol. 105, 923-932.

Chelh, I., Meunier, B., Picard, B., Reecy, J.M., Chevalier, C., Hocquette, J.F., Cassar-Malek, I., 2009. Molecular profiles of Quadriceps muscle in myostatin-null mice reveal PI3K and apoptotic pathways as myostatin targets. BMC Genomics 10, 196.

Chen, J., Sadowski, H.B., Kohanski, R.A., Wang, L.H., 1997. Stat5 is a physiological substrate of the insulin receptor. Proc. Natl. Acad. Sci. U. S. A. 94, 2295-2300.

Chinsomboon, J., Ruas, J., Gupta, R.K., Thom, R., Shoag, J., Rowe, G.C., Sawada, N., Raghuram, S., Arany, Z., 2009. The transcriptional coactivator PGC-1alpha mediates exercise-induced angiogenesis in skeletal muscle. Proc. Natl. Acad. Sci. U. S. A. 106, 21401-21406. 
Coffey, V.G., Shield, A., Canny, B.J., Carey, K.A., Cameron-Smith, D., Hawley, J.A., 2006.

Interaction of contractile activity and training history on mRNA abundance in skeletal muscle from trained athletes. Am. J. Physiol. Endocrinol. Metab. 290, E849-55.

Colussi, C., Banfi, C., Brioschi, M., Tremoli, E., Straino, S., Spallotta, F., Mai, A., Rotili, D., Capogrossi, M.C., Gaetano, C., 2010. Proteomic profile of differentially expressed plasma proteins from dystrophic mice and following suberoylanilide hydroxamic acid treatment. Proteomics Clin. Appl. 4, 71-83.

Dennis, G.,Jr, Sherman, B.T., Hosack, D.A., Yang, J., Gao, W., Lane, H.C., Lempicki, R.A., 2003. DAVID: Database for Annotation, Visualization, and Integrated Discovery. Genome Biol. 4, P3.

Egan, B., Zierath, J.R., 2013. Exercise metabolism and the molecular regulation of skeletal muscle adaptation. Cell. Metab. 17, 162-184.

Evans, N.P., Misyak, S.A., Robertson, J.L., Bassaganya-Riera, J., Grange, R.W., 2009.

Dysregulated intracellular signaling and inflammatory gene expression during initial disease onset in Duchenne muscular dystrophy. Am. J. Phys. Med. Rehabil. 88, 502-522.

Even, P.C., Decrouy, A., Chinet, A., 1994. Defective regulation of energy metabolism in mdxmouse skeletal muscles. Biochem. J. 304 ( Pt 2), 649-654.

Frigeri, A., Nicchia, G.P., Repetto, S., Bado, M., Minetti, C., Svelto, M., 2002. Altered aquaporin-4 expression in human muscular dystrophies: a common feature? FASEB J. 16, 1120-1122.

Grange, R.W., Call, J.A., 2007. Recommendations to define exercise prescription for Duchenne muscular dystrophy. Exerc. Sport Sci. Rev. 35, 12-17.

Hara, H., Nolan, P.M., Scott, M.O., Bucan, M., Wakayama, Y., Fischbeck, K.H., 2002. Running endurance abnormality in mdx mice. Muscle Nerve 25, 207-211.

Hoogaars, W.M., Mouisel, E., Pasternack, A., Hulmi, J.J., Relizani, K., Schuelke, M., Schirwis, E., Garcia, L., Ritvos, O., Ferry, A., 't Hoen, P.A., Amthor, H., 2012. Combined effect of AAV-U7induced dystrophin exon skipping and soluble activin Type IIB receptor in mdx mice. Hum. Gene Ther. 23, 1269-1279.

Hui, X., Zhu, W., Wang, Y., Lam, K.S., Zhang, J., Wu, D., Kraegen, E.W., Li, Y., Xu, A., 2009. Major urinary protein-1 increases energy expenditure and improves glucose intolerance through enhancing mitochondrial function in skeletal muscle of diabetic mice. J. Biol. Chem. 284, 1405014057.

Hulmi, J.J., Oliveira, B.M., Silvennoinen, M., Hoogaars, W.M., Ma, H., Pierre, P., Pasternack, A., Kainulainen, H., Ritvos, O., 2013a. Muscle protein synthesis, mTORC1/MAPK/Hippo signaling, and capillary density are altered by blocking of myostatin and activins. Am. J. Physiol. Endocrinol. Metab. 304, E41-50.

Hulmi, J.J., Oliveira, B.M., Silvennoinen, M., Hoogaars, W.M., Pasternack, A., Kainulainen, H., Ritvos, O., 2013b. Exercise restores decreased physical activity levels and increases markers of autophagy and oxidative capacity in myostatin/activin-blocked mdx mice. Am. J. Physiol.

Endocrinol. Metab. 305, E171-82. 
Jansen, M., van Alfen, N., Geurts, A.C., de Groot, I.J., 2013. Assisted bicycle training delays functional deterioration in boys with duchenne muscular dystrophy: the randomized controlled trial "no use is disuse". Neurorehabil. Neural Repair 27, 816-827.

Kainulainen, H., Hulmi, J.J., Kujala, U.M., 2013. Potential role of branched-chain amino Acid catabolism in regulating fat oxidation. Exerc. Sport Sci. Rev. 41, 194-200.

Kalista, S., Schakman, O., Gilson, H., Lause, P., Demeulder, B., Bertrand, L., Pende, M., Thissen, J.P., 2012. The type 1 insulin-like growth factor receptor (IGF-IR) pathway is mandatory for the follistatin-induced skeletal muscle hypertrophy. Endocrinology 153, 241-253.

Kallio, M.A., Tuimala, J.T., Hupponen, T., Klemela, P., Gentile, M., Scheinin, I., Koski, M., Kaki, J., Korpelainen, E.I., 2011. Chipster: user-friendly analysis software for microarray and other highthroughput data. BMC Genomics 12, 507-2164-12-507.

Kanehisa, M., Araki, M., Goto, S., Hattori, M., Hirakawa, M., Itoh, M., Katayama, T., Kawashima, S., Okuda, S., Tokimatsu, T., Yamanishi, Y., 2008. KEGG for linking genomes to life and the environment. Nucleic Acids Res. 36, D480-4.

Kivela, R., Silvennoinen, M., Lehti, M., Rinnankoski-Tuikka, R., Purhonen, T., Ketola, T., Pullinen, K., Vuento, M., Mutanen, N., Sartor, M.A., Reunanen, H., Koch, L.G., Britton, S.L., Kainulainen, H., 2010. Gene expression centroids that link with low intrinsic aerobic exercise capacity and complex disease risk. FASEB J. 24, 4565-4574.

Klover, P., Hennighausen, L., 2007. Postnatal body growth is dependent on the transcription factors signal transducers and activators of transcription $5 \mathrm{a} / \mathrm{b}$ in muscle: a role for autocrine/paracrine insulin-like growth factor I. Endocrinology 148, 1489-1497.

Koenig, M., Hoffman, E.P., Bertelson, C.J., Monaco, A.P., Feener, C., Kunkel, L.M., 1987. Complete cloning of the Duchenne muscular dystrophy (DMD) cDNA and preliminary genomic organization of the DMD gene in normal and affected individuals. Cell 50, 509-517.

Kujala, U.M., Kaprio, J., Sarna, S., Koskenvuo, M., 1998. Relationship of leisure-time physical activity and mortality: the Finnish twin cohort. JAMA 279, 440-444.

Kujala, U.M., Makinen, V.P., Heinonen, I., Soininen, P., Kangas, A.J., Leskinen, T.H., Rahkila, P., Wurtz, P., Kovanen, V., Cheng, S., Sipila, S., Hirvensalo, M., Telama, R., Tammelin, T., Savolainen, M.J., Pouta, A., O'Reilly, P.F., Mantyselka, P., Viikari, J., Kahonen, M., Lehtimaki, T., Elliott, P., Vanhala, M.J., Raitakari, O.T., Jarvelin, M.R., Kaprio, J., Kainulainen, H., Ala-Korpela, M., 2013. Long-term leisure-time physical activity and serum metabolome. Circulation 127, 340348.

Kuznetsov, A.V., Winkler, K., Wiedemann, F.R., von Bossanyi, P., Dietzmann, K., Kunz, W.S., 1998. Impaired mitochondrial oxidative phosphorylation in skeletal muscle of the dystrophindeficient mdx mouse. Mol. Cell. Biochem. 183, 87-96.

Landisch, R.M., Kosir, A.M., Nelson, S.A., Baltgalvis, K.A., Lowe, D.A., 2008. Adaptive and nonadaptive responses to voluntary wheel running by mdx mice. Muscle Nerve 38, 1290-1303. 
LeBrasseur, N.K., Schelhorn, T.M., Bernardo, B.L., Cosgrove, P.G., Loria, P.M., Brown, T.A., 2009. Myostatin inhibition enhances the effects of exercise on performance and metabolic outcomes in aged mice. J. Gerontol. A Biol. Sci. Med. Sci. 64, 940-948.

Lee, S.J., Reed, L.A., Davies, M.V., Girgenrath, S., Goad, M.E., Tomkinson, K.N., Wright, J.F., Barker, C., Ehrmantraut, G., Holmstrom, J., Trowell, B., Gertz, B., Jiang, M.S., Sebald, S.M., Matzuk, M., Li, E., Liang, L.F., Quattlebaum, E., Stotish, R.L., Wolfman, N.M., 2005. Regulation of muscle growth by multiple ligands signaling through activin type II receptors. Proc. Natl. Acad. Sci. U. S. A. 102, 18117-18122.

Leskinen, T., Rinnankoski-Tuikka, R., Rintala, M., Seppanen-Laakso, T., Pollanen, E., Alen, M., Sipila, S., Kaprio, J., Kovanen, V., Rahkila, P., Oresic, M., Kainulainen, H., Kujala, U.M., 2010. Differences in muscle and adipose tissue gene expression and cardio-metabolic risk factors in the members of physical activity discordant twin pairs. PLoS One 5, 10.1371/journal.pone.0012609.

Ljubicic, V., Burt, M., Jasmin, B.J., 2014. The therapeutic potential of skeletal muscle plasticity in Duchenne muscular dystrophy: phenotypic modifiers as pharmacologic targets. FASEB J. 28, 548568.

Ljubicic, V., Miura, P., Burt, M., Boudreault, L., Khogali, S., Lunde, J.A., Renaud, J.M., Jasmin, B.J., 2011. Chronic AMPK activation evokes the slow, oxidative myogenic program and triggers beneficial adaptations in mdx mouse skeletal muscle. Hum. Mol. Genet. 20, 3478-3493.

Matsakas, A., Macharia, R., Otto, A., Elashry, M.I., Mouisel, E., Romanello, V., Sartori, R., Amthor, H., Sandri, M., Narkar, V., Patel, K., 2012. Exercise training attenuates the hypermuscular phenotype and restores skeletal muscle function in the myostatin null mouse. Exp. Physiol. 97, 125140.

Matsakas, A., Mouisel, E., Amthor, H., Patel, K., 2010. Myostatin knockout mice increase oxidative muscle phenotype as an adaptive response to exercise. J. Muscle Res. Cell. Motil. 31, 111-125.

McPherron, A.C., Lee, S.J., 1997. Double muscling in cattle due to mutations in the myostatin gene. Proc. Natl. Acad. Sci. U. S. A. 94, 12457-12461.

Mero, A.A., Hulmi, J.J., Salmijarvi, H., Katajavuori, M., Haverinen, M., Holviala, J., Ridanpaa, T., Hakkinen, K., Kovanen, V., Ahtiainen, J.P., Selanne, H., 2013. Resistance training induced increase in muscle fiber size in young and older men. Eur. J. Appl. Physiol. 113, 641-650.

Mikus, C.R., Boyle, L.J., Borengasser, S.J., Oberlin, D.J., Naples, S.P., Fletcher, J., Meers, G.M., Ruebel, M., Laughlin, M.H., Dellsperger, K.C., Fadel, P.J., Thyfault, J.P., 2013. Simvastatin impairs exercise training adaptations. J. Am. Coll. Cardiol.

Moens, P., Baatsen, P.H., Marechal, G., 1993. Increased susceptibility of EDL muscles from mdx mice to damage induced by contractions with stretch. J. Muscle Res. Cell. Motil. 14, 446-451.

Mootha, V.K., Lindgren, C.M., Eriksson, K.F., Subramanian, A., Sihag, S., Lehar, J., Puigserver, P., Carlsson, E., Ridderstrale, M., Laurila, E., Houstis, N., Daly, M.J., Patterson, N., Mesirov, J.P., Golub, T.R., Tamayo, P., Spiegelman, B., Lander, E.S., Hirschhorn, J.N., Altshuler, D., Groop, L.C., 2003. PGC-1alpha-responsive genes involved in oxidative phosphorylation are coordinately downregulated in human diabetes. Nat. Genet. 34, 267-273. 
Nicchia, G.P., Cogotzi, L., Rossi, A., Basco, D., Brancaccio, A., Svelto, M., Frigeri, A., 2008. Expression of multiple AQP4 pools in the plasma membrane and their association with the dystrophin complex. J. Neurochem. 105, 2156-2165.

Personius, K.E., Jayaram, A., Krull, D., Brown, R., Xu, T., Han, B., Burgess, K., Storey, C., Shah, B., Tawil, R., Welle, S., 2010. Grip force, EDL contractile properties, and voluntary wheel running after postdevelopmental myostatin depletion in mice. J. Appl. Physiol. 109, 886-894.

Pistilli, E.E., Bogdanovich, S., Goncalves, M.D., Ahima, R.S., Lachey, J., Seehra, J., Khurana, T., 2011. Targeting the activin type IIB receptor to improve muscle mass and function in the mdx mouse model of Duchenne muscular dystrophy. Am. J. Pathol. 178, 1287-1297.

Ploquin, C., Chabi, B., Fouret, G., Vernus, B., Feillet-Coudray, C., Coudray, C., Bonnieu, A., Ramonatxo, C., 2012. Lack of myostatin alters intermyofibrillar mitochondria activity, unbalances redox status, and impairs tolerance to chronic repetitive contractions in muscle. Am. J. Physiol. Endocrinol. Metab. 302, E1000-8.

Rahimov, F., King, O.D., Warsing, L.C., Powell, R.E., Emerson, C.P.,Jr, Kunkel, L.M., Wagner, K.R., 2011. Gene expression profiling of skeletal muscles treated with a soluble activin type IIB receptor. Physiol. Genomics 43, 398-407.

Rantalainen, T., Silvennoinen, M., Kainulainen, H., Sievanen, H., 2011. Vertical ground reaction force measurements and video measurements provide comparable estimates of distance moved by mice during artificial light and dark periods. J. Neurosci. Methods 197, 104-108.

Relizani, K., Mouisel, E., Giannesini, B., Hourde, C., Patel, K., Morales Gonzalez, S., Julich, K., Vignaud, A., Pietri-Rouxel, F., Fortin, D., Garcia, L., Blot, S., Ritvos, O., Bendahan, D., Ferry, A., Ventura-Clapier, R., Schuelke, M., Amthor, H., 2014. Blockade of ActRIIB Signaling Triggers Muscle Fatigability and Metabolic Myopathy. Mol. Ther. 22, 1423-1433.

Silvennoinen, M., Rantalainen, T., Kainulainen, H., 2014. Validation of a method to measure total spontaneous physical activity of sedentary and voluntary running mice. J. Neurosci. Methods 235C, $51-58$.

Smyth, G.K., 2004. Linear models and empirical bayes methods for assessing differential expression in microarray experiments. Stat. Appl. Genet. Mol. Biol. 3, Article3.

Smyth, G.K., Speed, T., 2003. Normalization of cDNA microarray data. Methods 31, 265-273.

Subramanian, A., Tamayo, P., Mootha, V.K., Mukherjee, S., Ebert, B.L., Gillette, M.A., Paulovich, A., Pomeroy, S.L., Golub, T.R., Lander, E.S., Mesirov, J.P., 2005. Gene set enrichment analysis: a knowledge-based approach for interpreting genome-wide expression profiles. Proc. Natl. Acad. Sci. U. S. A. 102, 15545-15550.

Sugden, M.C., Holness, M.J., 2003. Recent advances in mechanisms regulating glucose oxidation at the level of the pyruvate dehydrogenase complex by PDKs. Am. J. Physiol. Endocrinol. Metab. 284, E855-62.

Timmons, J.A., Larsson, O., Jansson, E., Fischer, H., Gustafsson, T., Greenhaff, P.L., Ridden, J., Rachman, J., Peyrard-Janvid, M., Wahlestedt, C., Sundberg, C.J., 2005. Human muscle gene 
expression responses to endurance training provide a novel perspective on Duchenne muscular dystrophy. FASEB J. 19, 750-760.

Wagenmakers, A.J., 1998. Muscle amino acid metabolism at rest and during exercise: role in human physiology and metabolism. Exerc. Sport Sci. Rev. 26, 287-314.

Webster, C., Silberstein, L., Hays, A.P., Blau, H.M., 1988. Fast muscle fibers are preferentially affected in Duchenne muscular dystrophy. Cell 52, 503-513.

Welle, S., Bhatt, K., Pinkert, C.A., 2006. Myofibrillar protein synthesis in myostatin-deficient mice. Am. J. Physiol. Endocrinol. Metab. 290, E409-15.

Zhao, B., Li, E.J., Wall, R.J., Yang, J., 2009. Coordinated patterns of gene expressions for adult muscle build-up in transgenic mice expressing myostatin propeptide. BMC Genomics 10, 3052164-10-305.

Zhou, X., Wang, J.L., Lu, J., Song, Y., Kwak, K.S., Jiao, Q., Rosenfeld, R., Chen, Q., Boone, T., Simonet, W.S., Lacey, D.L., Goldberg, A.L., Han, H.Q., 2010. Reversal of cancer cachexia and muscle wasting by ActRIIB antagonism leads to prolonged survival. Cell 142, 531-543.

\section{ACKNOWLEDGEMENTS}

We would like to thank Tuuli Nissinen, Hongqiang Ma, Kaisa-Leena Tulla, Mervi Matero, Simon Walker, Ritva Taipale, Jaakko Hentilä, Juho Hyödynmaa, Timo Rantalainen, Risto Puurtinen, Aila Ollikainen, Paavo Rahkila, Eliisa Kiukkanen and Mia Horttanainen for their help.

\section{GRANTS}

This work was supported by Academy of Finland (Decision No. 137787 to JJH and decision no. 134117 to RA) and the Finnish Cultural Foundation and Paulo Foundation (HK).

\section{DISCLOSURES}

No conflicts of interest, financial or otherwise, are declared by the authors.

\section{AUTHOR CONTRIBUTIONS}

$\mathrm{JJH}$ and BMO carried out the in vivo experiments. MS assisted in the in vivo experiments and isolated muscles. JJH and HK drafted the manuscript with the help from KGP, UMK, RA and 
PACH. JJH, KGP and MS carried out the analyses into which HK, RA, PACH, JS and MN also provided assistance. JJH, HK and OR designed the study with help from MS and PACH. AP and OR prepared the recombinant sActRIIB-Fc used in the study. All authors read and approved the final manuscript.

\section{Figure legends}

Figure 1. RNA, DNA and protein concentrations in gastrocnemius muscle of mdx mice treated with SActRIIB-Fc and exercise alone or in combination and in comparison to wild type controls. A and C-F) RNA, DNA and protein concentrations per total muscle wet weight or per DNA or RNA in gastrocnemius muscle. B) Ribosomal RNA shown using agarose gel electrophoresis of total RNA. For that, total RNA was extracted as described in methods and subjected to electrophoresis through an agarose gel. A representative gel is shown. $28 \mathrm{~S}$ is $28 \mathrm{~S}$ ribosomal RNA, $18 \mathrm{~S}$ is $18 \mathrm{~S}$ ribosomal $\mathrm{RNA} . \mathrm{mdx}=$ dystrophic mice, $\mathrm{wt}=$ wild type mice. $\mathrm{PBS}=$ placebo control injection, AcR $=$ sActRIIB-Fc injection, Run $=$ wheel running. ${ }^{*}=\mathrm{P}<0.05$ vs. PBS injected mdx mice.

Figure 2. The gene sets of aerobic metabolism are modulated in the skeletal muscle of $\mathbf{m d x}$ mice and regulated towards wild type controls with exercise alone and in combination with sActRIIB-Fc. Gene Set Enrichment Analysis (GSEA) (Subramanian et al 2005) was conducted from microarray of gastrocnemius muscle. Different gene set results are shown in A-H. A) Electron transport chain, B) oxidative phosphorylation, C) TCA cycle (tricarboxylic acid acid cycle), D) BCAA degradation (degradation of branched chain amino acids valine, leucine and isoleucine), E) fatty acid metabolism, F) peroxisome, G) metabolism of lipids and lipoproteins, H) glucose regulation of insulin secretion. NES = normalized enrichment score in GSEA. Positive and negative enrichment scores denote a large number of genes upregulated or downregulated, respectively, in the given gene set. $*=\mathrm{FDR}<0.05, * *=\mathrm{FDR}<0.01, * * *=\mathrm{FDR}<0.001$. If FDR $>0.05$, the exact FDR-values are shown. $\mathrm{mdx}-\mathrm{Cont}$ or $\mathrm{wt}-\mathrm{Cont}=\mathrm{mdx}$ mice or wild type mice, respectively, injected PBS control. AcR $=$ sActRIIB-Fc injection, Run $=$ wheel running. Exact effect size scores and FDR-values for these and other comparisons are in (Supplemental Table S2).

Figure 3. Heat map of lipid metabolism pathway (SP_PIR_KEYWORDS). For this, genes with $\geq 1.5$ fold change differences between the groups were loaded to DAVID. The difference in lipid metabolism between $\mathrm{mdx}$ and wild type mice is at least partially corrected with running as most of the running mice show more similar profile with the wild type mice than the other groups. mdxCont or wt-Cont $=\mathrm{mdx}$ mice or wild type mice, respectively, injected PBS control. AcR = sActRIIB-Fc injection, Run = wheel running. 
Figure 4. Protein results after 7 weeks. A) Phosrphorylated (p) STAT5 and total STAT5, B) MUP, C) PDK4, D) UCP3, E) pERK 1/2 and total ERK 1/2, F) FASN. G) Representative blots. mdx = dystrophic mice, $\mathrm{wt}=$ wild type mice. In the representative blots, $\mathrm{mdx}-\mathrm{Cont}=\mathrm{mdx}$ mice injected PBS control, $m d x-R u n=m d x$ mice with wheel running, $m d x-A c R=m d x$ mice injected with sActRIIB-Fc, mdx-AcR Run = mdx mice treated with sActRIIB-Fc and wheel running, wt-Cont $=$ wild type mice treated with PBS control. The data is presented as relative to PBS injected mdx mice. In MUP blot, $+=$ positive control lysate. Band at $\sim 20 \mathrm{kDa}$ also seen in positive control was quantified. * alone indicates statistical difference $(\mathrm{p}<0.05)$ compared to PBS injected mdx mice or to another group if above a marked line. The results are corrected to the mean of Ponceau $\mathrm{S}$ and GAPDH value.

Figure 5. Home-cage physical activity before and during the 7-wk period. A) Activity time and B) activity index using force plate measurements. $\mathrm{mdx}-\mathrm{Cont}=\mathrm{mdx}$ mice injected PBS control, $m d x-R u n=m d x$ mice injected PBS and treated with wheel running, $m d x-A c R=m d x$ mice injected with sActRIIB-Fc, mdx-AcR Run $=m d x$ mice treated with sActRIIB-Fc and wheel running, wt-Cont $=$ wild type mice treated with PBS control.* indicates statistical difference $(p<0.05)$ to wild type control mice at each time-point.

\section{Tables}

Table 1. The number of genes modulated by treatments in mdx mice closer to (more than $50 \%$ towards) healthy wild type controls. For this analysis, we selected only the gene probes with average expression level significant at a level $\mathrm{P}<0.05$ over background. From the genes represented by multiple probes, the probe with the highest fold-change ratio was selected. The calculation of genes toward or away from controls was conducted for all these probes using a $50 \%$ distance criteria using self-developed Macro in Microsoft Excel. Thus, no change means that still after the treatment, the average value of the gene in the group is closer to mdx mice than wild type control. Instead, positive and negative means that after the treatment the result is more than $50 \%$ toward or more than $50 \%$ away from healthy wild type mouse, respectively.

\section{Comparison to wild type control}

mdx sActRIIB-Fc

mdx Run

mdx ActRIIB-Fc Run

$\begin{array}{lll}\text { Positive } & \text { Negative } & \text { No change } \\ 1696 & 1553 & 5376 \\ 1746 & 1335 & 5544 \\ 2008 & 1689 & 4928\end{array}$


A)

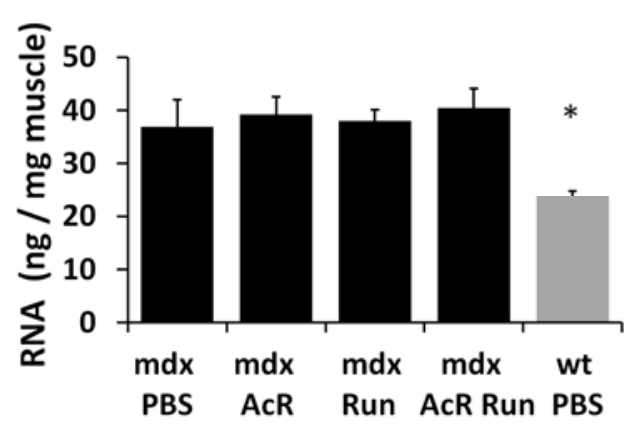

B)

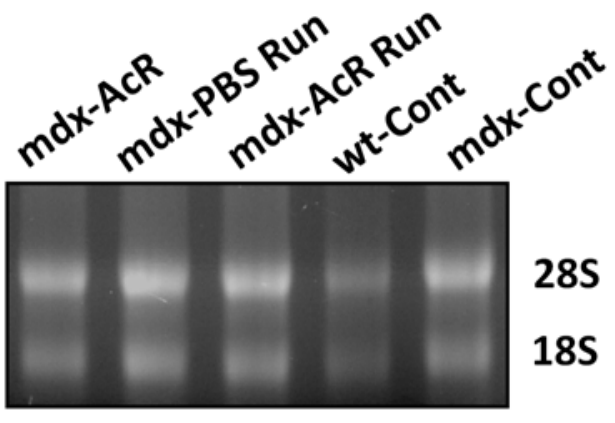

C)

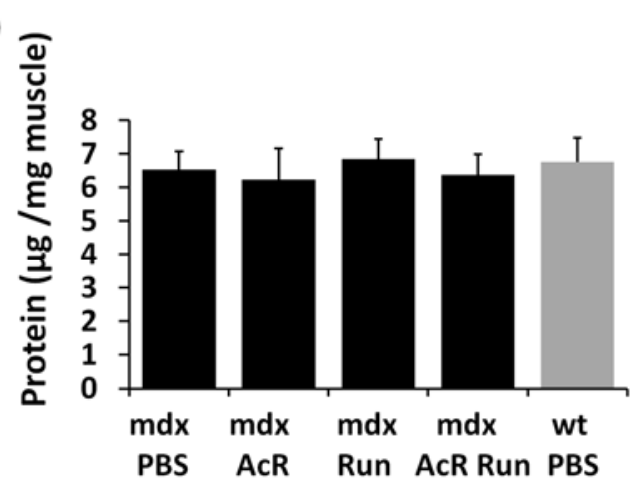

E)

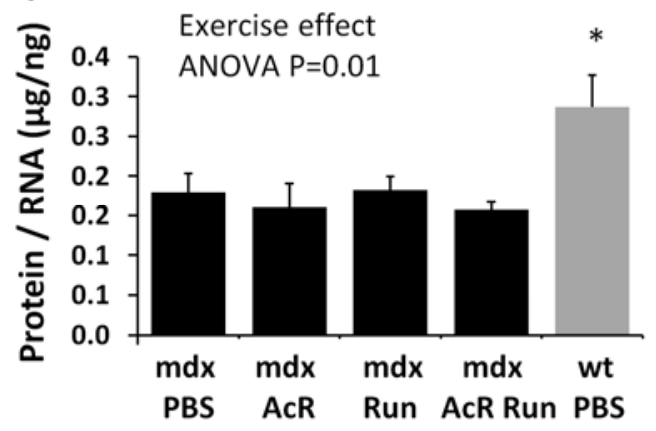

D)

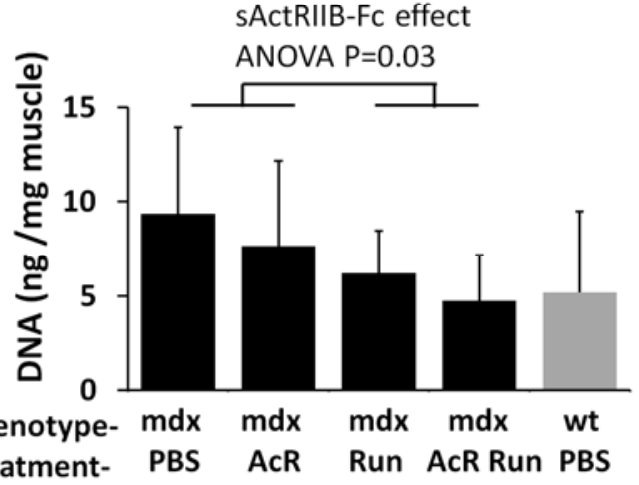

F)

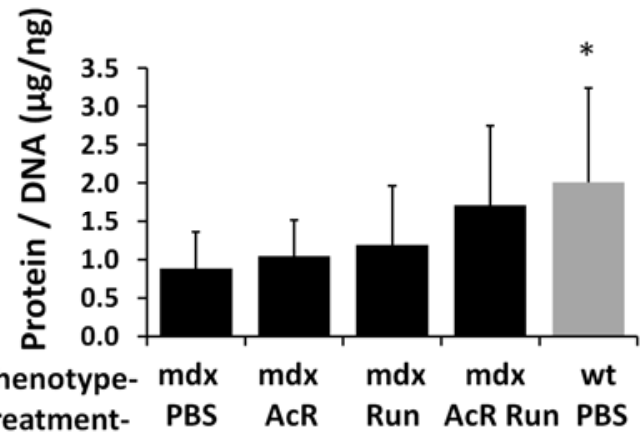



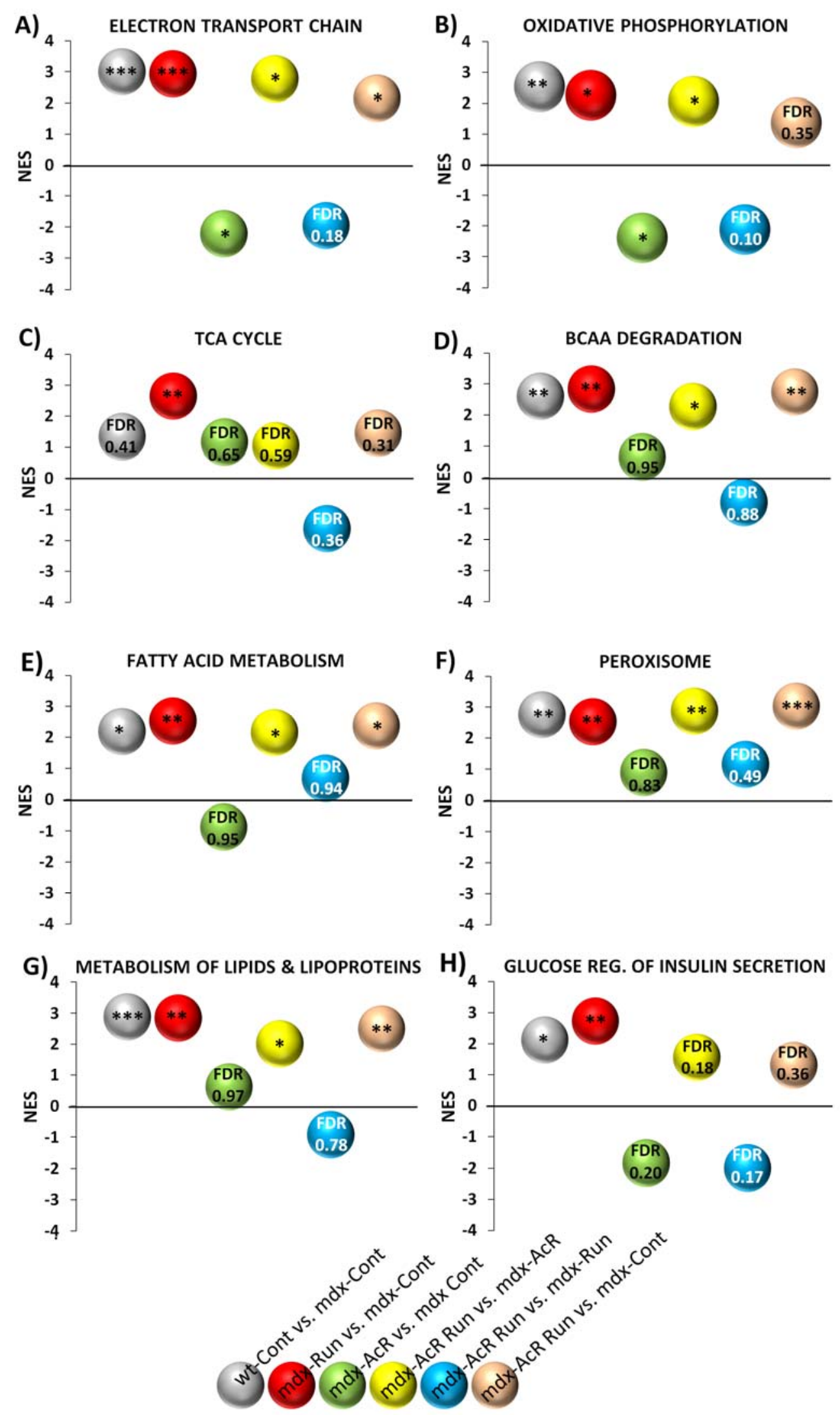


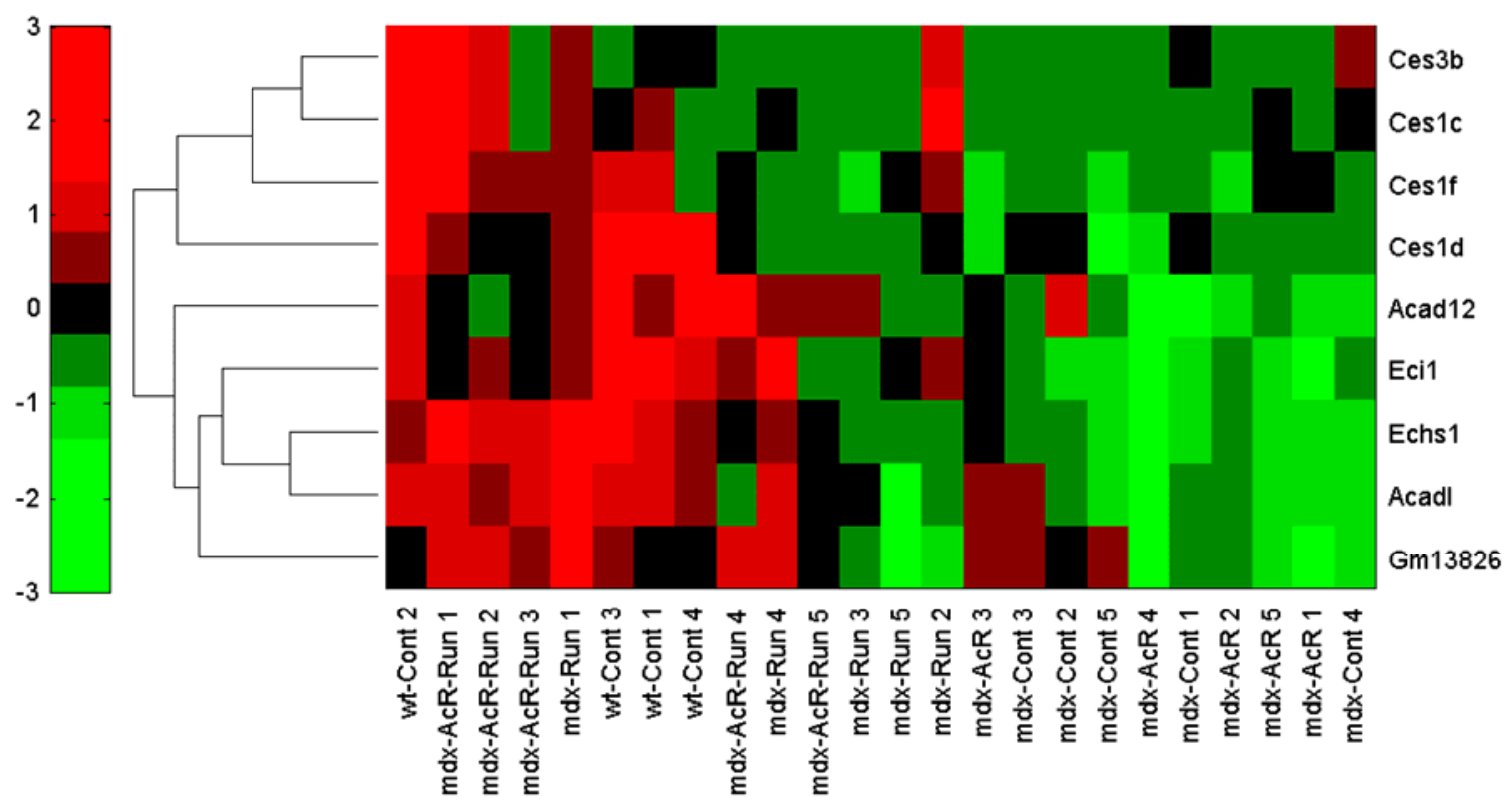




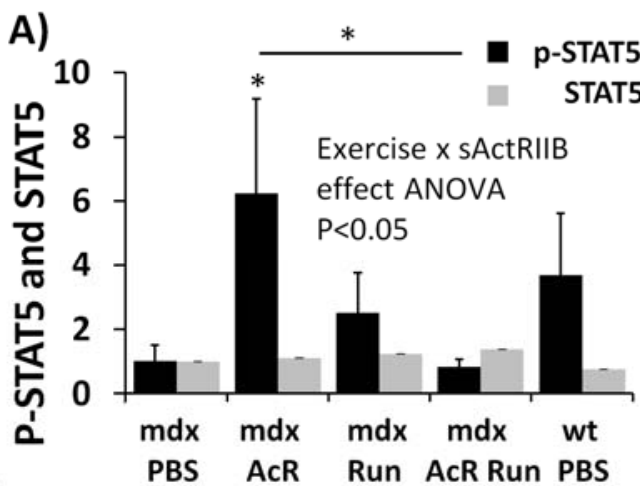

B)

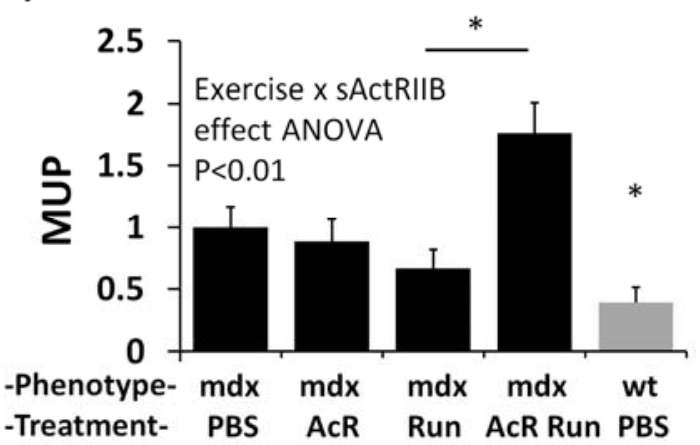

C)

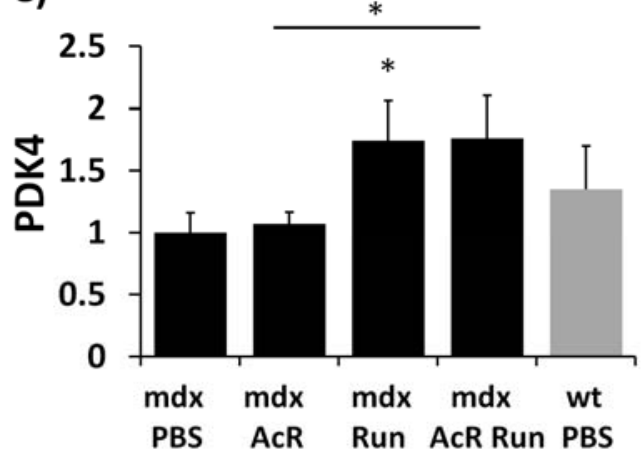

E)

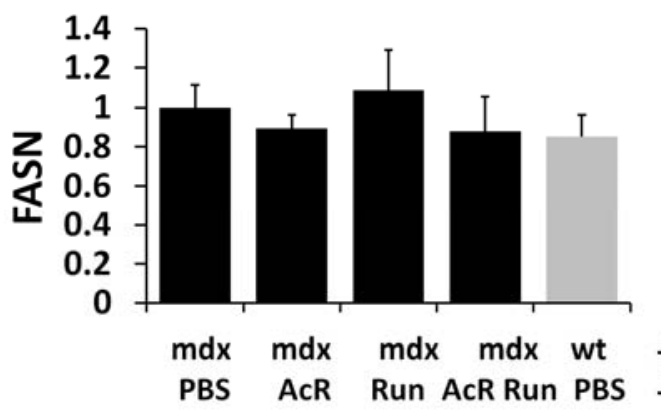

D)

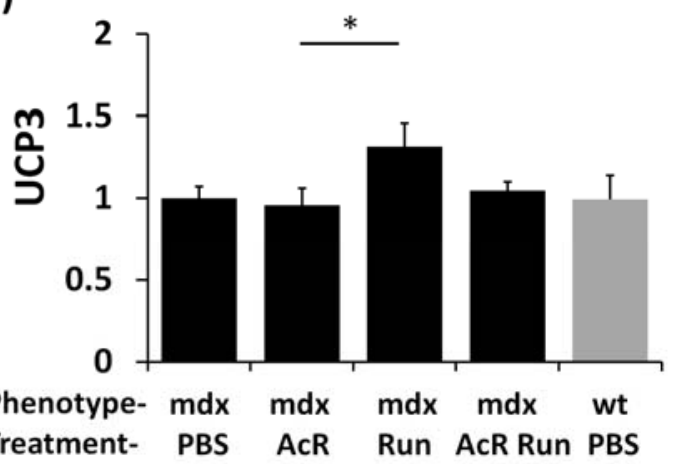

G)

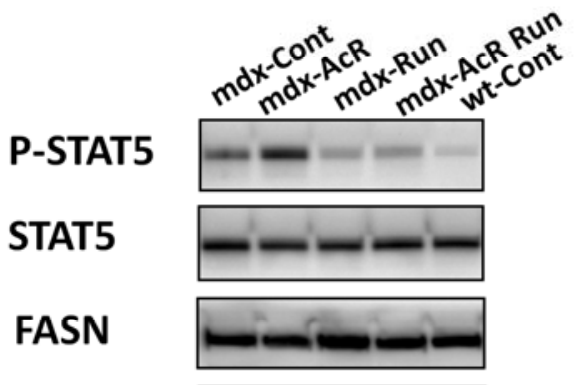

F)

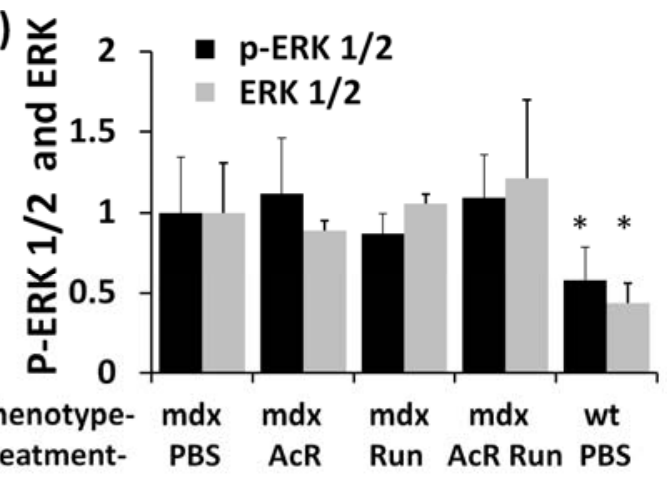

pERK 1/2

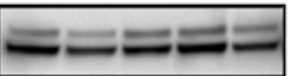

ERK 1/2

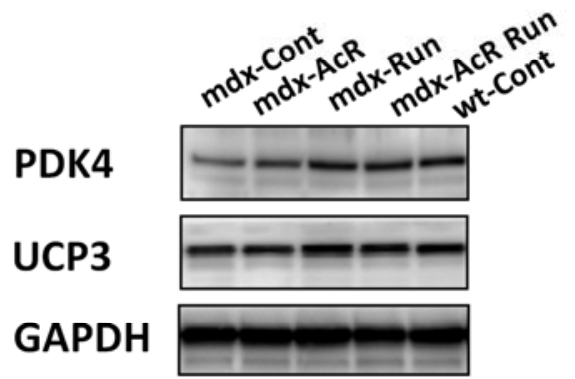

GAPDH

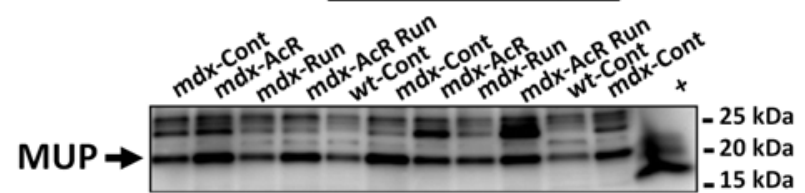


$\square$ mdx-Cont $\square$ mdx-AcR $\square$ mdx-Run $\square$ mdx-AcR Run $\square$ wt-Cont

A)

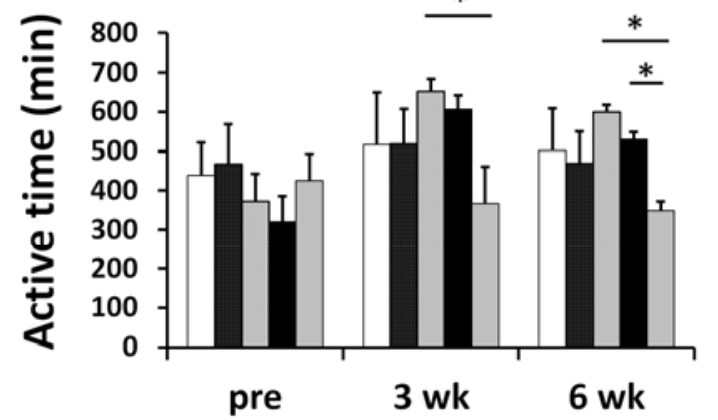

B)

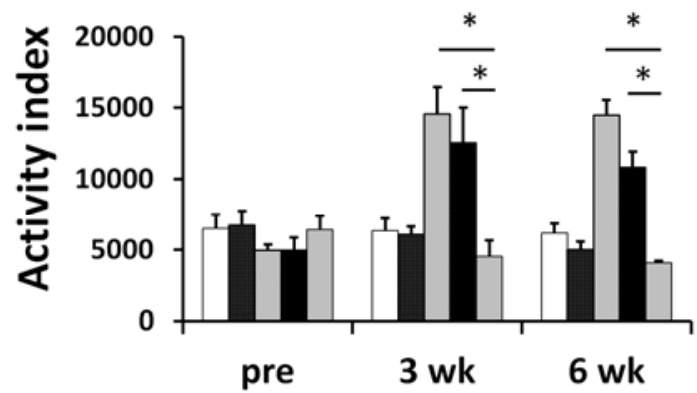

